\title{
IgG4 Related Peri-aortitis: A Case Diagnosed in Combined Military Hospital, Dhaka
}

\author{
Razzak MA ${ }^{1}$, Alam SZ2 ${ }^{2}$, Nasrin $\mathrm{S}^{3}$, Rahman QAA ${ }^{4}$ \\ DOI: https://doi.org/10.3329/jafmc.v14i2.45914
}

\begin{abstract}
IgG4 related disease is a multi-organ immune-mediated condition which links many disorders previously regarded as isolated, a single-organ disease without any known underlying systemic condition. It may mimic anymalignant, infectious and inflammatory disorders. It was recognized as a unified entity only 10 years ago. Histopathology is the key to diagnosis. The three central pathology features of lgG4 related disease are lymphoplasmacytic infiltration, stroriform fibrosis, and obliterative arteritis. It is important to promptly diagnose lgG4 related disease because this disease commonly manifests as swelling of the affected organs, which must be differentiated from true neoplasms. Periaortitis or periarteritis is one of the clinical features of lgG4 related diseases. IgG4 related arterial lesions occur mainly in the aorta and its main branches and radiologically characterized by homogeneous arterial wall thickening corresponding to pathological features of $\mathrm{lgG} 4$ related sclerosing inflammation in the adventitia. There are 5 types of $\lg$ G4 related peri-aortitis, out of which Type 2 is most common. IgG4 related peri-aortitis generally respond to glucocorticoids in its inflammatory stage, but recurrent or refractory cases are common. Greater awareness of this disease is needed to ensure earlier diagnoses to prevent severe organ damage. A 38-year-old male was diagnosed as Type 2 lgG4 related periaortitis 2 years ago in $\mathrm{CMH}$, Dhaka. He was treated with oral steroid and Azathioprine with a rewarding outcome.
\end{abstract}

Key-words: IgG4, Aortitis, Peri-aortitis, Immune-mediated.

\section{Introduction}

lgG4 related disease (IgG4-RD), formerly known as IgG4related systemic disease, is a chronic inflammatory condition characterized by tissue infiltration with lymphocytes and lgG4 secreting plasma cells, various degrees of fibrosis (scarring) and usually prompt response to oral steroids. In approximately $51-70 \%$ of people with this disease, serum lgG4 concentrations are elevated during an acute phase ${ }^{1-3}$. It is a relapsing-remitting disease associated with a tendency to mass forming, tissue destructive lesions in multiple sites, with a characteristic histopathological appearance in whichever site is involved. Inflammation and deposition of connective tissues in affected anatomical sites can lead to organ dysfunction or even organ failure if not treated ${ }^{4}$. IgG4-RD can involve one or multiple sites in the body, the sites involved can be affected at the same tissue or unrelated different tissue. It may affect the pancreas, biliary tree, liver, brain, nerves, eyes, heart, blood vessels, throat, airway, oesophagus, intestine, kidney, prostate, testes, thyroid, breast, pituitary gland, mediastinum, retro-peritoneum, meninges, peritoneum, pleura etc. Some common manifestations of this disease include Type I autoimmune pancreatitis, interstitial nephritis, Riedel's thyroiditis, inflammatory pseudotumor at various sites, mediastinal fibrosis, retroperitoneal fibrosis, prostatitis, orchitis and periaortitis or periarteritis ${ }^{5,6}$.

Aortitis means inflammation of aortic wall which is a rare but potentially life-threatening condition. It may be caused by trauma, viral or bacterial infection (notably syphilis) and certain immune disordersand connective tissue diseases. Chronic periaortitisis a rare disease, occurs usually in middleaged adult men. It includes a variety of conditions that have similar clinical histopathological findings and this probably represents a different manifestation of the same disease such as idiopathic retroperitoneal fibrosis, perianeurysmal retroperitoneal fibrosis and inflammatory abdominal aortic aneurysms. The three entities are characterized by advanced atherosclerosis of the abdominal aorta, adventitial and periadventitial inflammation, medial thinning and chronic retroperitoneal inflammatory process associated with varying degree of fibrotic reaction surrounding the abdominal aorta. Periaortitis may be an expression of the localimmune response to antigens such as oxidized low-density lipoproteins and ceroid in the atherosclerotic plaques of the abdominal aorta. Periaortitis mayalso be considered a manifestation of systemic autoimmune diseases suchas ankylosing spondylitis, rheumatoid arthritis and systemic lupus erythematosus. A far rare cause of periaortits is $\lg G 4$ related periaortitis ${ }^{7}$.

When IgG4-RD affects only the aorta, it is called isolated periaortitis. This is a very rare condition. In Department of Rheumatology, CMH Dhaka, we have diagnosed a case of lgG4 related periaortitis first time in Bangladesh on 25July 2017. This case report is published to generate awareness among the physicians regarding the early diagnosis and treatment of such patient.

1. Brig Gen Md Abdur Razzak, MBBS, MCPS, FCPS, APLAR Fellow in Rheumatology, Professor \& Head, Department of Medicine, AFMC, Dhaka (E-mail: razzakprm@yahoo.com) 2. Brig Gen Syed Zoherul Alam, MBBS, FCPS, Adviser Specialist \& Head, Department of Radiology and Imaging, CMH, Dhaka 3. Maj Shamima Nasrin, MBBS, Graded Specialist in Radiology, CMH, Savar 4. Dr Quazi Audry Arafat Rahman, MBBS, Assistant Registrar, Department of Medicine, AFMC, FCPS Part II Trainee, Kurmitola General Hospital, Dhaka. 


\section{Case Report}

Mr ' $X$ ' aged 38 years, a referred case from Combined Military Hospital (CMH)Savar was admitted to $\mathrm{CMH}$ Dhaka on 25 July 2017. He presented with dull aching pain in the lower abdomen with occasional radiation to groins for the last 3 months. He also complained of the passage of mucous mixed stool with fatigue and some weight loss for the same duration. There was no history of blood mixed micturition or stool, fever, vomiting. He had constant low back pain without any radiation. He was not responding to symptomatic treatment with analgesic and antispasmodic medications. Routine investigations like CBC, Urine and Stool R/E, Lipid profile, blood sugar, serum amylase, renal and liver functions were normal. But CRP was positive. Plain X-ray abdomen, KUB and L/S spine were unremarkable. USG of the whole abdomen revealed no abnormality.

Due to persistent symptom, CT-scan of the abdomen (Figure-1)was done which revealed long segment dilation of abdominal aorta extending from the origin of renal artery up to common iliac artery suggestive of inflammatory lesion. For further evaluation, CT angiogram (Figure-2) was done which showed circumferential wall thickening surrounding the same segment of the abdominal aorta with mild luminal narrowing suggestive of isolated periaortitis. Rest of the visualized portion of the abdominal aorta and its branches including both common iliac arteries were normal in outline and calibre. The Doppler ultrasound revealed similar findings.

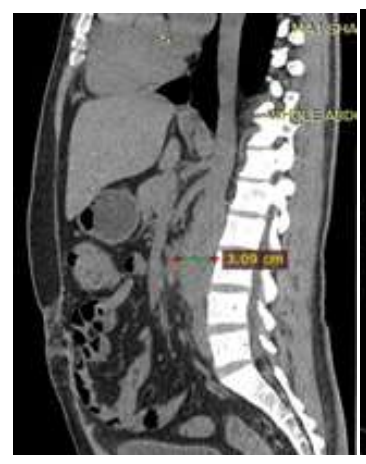

(a)

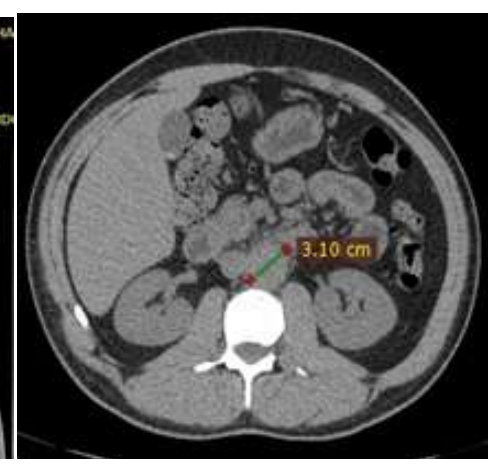

(b)
Figure-1: CT abdomen revealing long segment dilatation of abdominal aorta

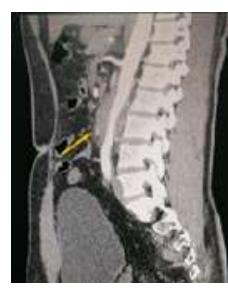

(a)

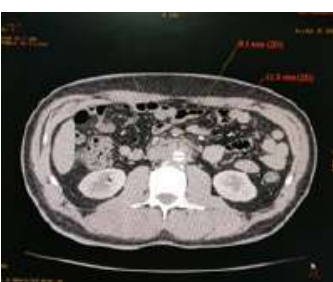

(b)

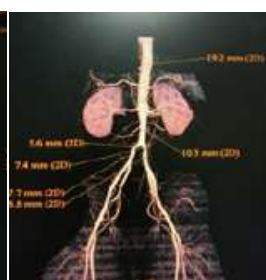

(c)
Figure-2: CT angiogram revealing circumferential wall thickening surrounding Abdominal Aorta
To find out the immunological cause of periaortitis, c-ANCA and $\mathrm{p}$-ANCA was done which were negative. Keeping in mind the association of Aortitis with Ankylosing Spondylitis and Syphilis HLA-B27 and VDRL was done; both of which were negative. Then keeping in mind, the strong suspicion of lgG4 related periaortitis, IgG level was done from ICDDR,B and the level was significantly high $(1529 \mathrm{mg} / \mathrm{dL}$; normal value $200-$ $400 \mathrm{mg} / \mathrm{dL}$ ). Thereby, the lgG4 level was done from India (test facility is not available in Bangladesh; therefore, the sample was sent to India through Apollo Hospital, Dhaka), which was significantly high $(548 \mathrm{mg} / \mathrm{dl}$; normal value 2-121 $\mathrm{mg} / \mathrm{dl})$. Finally, the patient was diagnosed as a case of $\mathrm{lgG} 4$ related periaortitis. The patient was treated with oral steroid and Azathioprine as immunosuppressive and steroid-sparing agent. The response to treatment was rewarding. The patient became symptom-free within a few weeks. Follow up CT scan (on 29/5/2018) showed regression of the lesion. The patient is on maintenance therapy and under regular checkup.

\section{Discussion}

Periaortitis and periarteritis are one of the clinical features of IgG4-RD. Such vascular manifestations are reported to be present in $10-30 \%$ of overall $\lg G 4-R D$, and they may also appear as an isolated lesion of lgG4-related disease. Like other types of large vessel vasculitis, IgG4-related vasculitis may cause significant clinical consequences ${ }^{8}$. IgG4 related periaortitis has a male predominance and ittends to occur in patients having the onset of IgG4-RD at an older age and in those with highly active disease states displaying elevated serum IgG, IgG4, Circulating Immune Complexes (CIC), and serum soluble interleukin-2 receptor(sIL2R). Thus, we consider a high IgG4-RD activity state to most strongly influence the onset of periaortitis/periarteritis. Hypertension and smoking were not risk factors in some studies ${ }^{9}$. Some patients with IgG4-related periaortitis/periarteritis presents with pain (chest, abdomen, back, and groin), dyspnea and oedema, but most of the symptoms are non-specific ${ }^{8}$. IgG4-related vascular lesions are most frequently reported in the aorta, most commonly in the infra-renal portion of the abdominal aorta. In addition, medium-sized blood vessels, such as the celiac, mesenteric, iliac, coronary, pulmonary, and vertebral arteries have been reported as potential targets of IgG4-related periaortitis/ periarteritis. Involvement of other medium-sized vessels has also been reported such as superior/inferior mesenteric arteries, common iliac artery, splenic arteries, carotid artery etc10.Periaortitis/periarteritis could be broadly classified into five types 11 and the most prevalent is Type2 in which our case belongs to.

The diagnosis of IgG4-related periaortitis/periarteritis seems to have been made based on different diagnostic criteria in reported studies. For example, some studies reported concomitant vascular lesions in patients with known lgG4related disease in non-vascular organs, while others reported the histopathological diagnosis of vascular involvement in lgG4-related disease. In addition, some studies report only 
definitive cases of IgG4-related periaortitis/periarteritis, while others also describe probable or possible cases ${ }^{11}$. IgG4-related cardiovascular disorders can severely affect patient prognosis, various imaging techniques including echocardiography, $\mathrm{CT}$, 18F-FDG-PET, cardiovascular magnetic resonance (CMR), and cardiac catheterization have been successfully adopted for early disease detection and follow-up ${ }^{12}$. Characteristic computed tomography (CT) findings of IgG4-related periaortitis/ periarteritis include a severely thickened aortic/arterial wall, which is involved circumferentially and homogeneously enhanced at the delayed phase of dynamic contrast-enhanced CT. A high-density thin layer corresponding to the intimamedia complex is sometimes seen. Characteristic magnetic resonance imaging (MRI) findings have not been evaluated ${ }^{10}$. Studies have shown that most patients who receive corticosteroids show improvements in wall thickening after therapy, although few cases may exhibit worsening of luminal dilatation ${ }^{13}$. Some studies have described that the luminal diameters of affected vessels were not changed or dilated, while others reported rupture after corticosteroid therapy. Other drugs like Azathioprine, Methotrexate, Cyclophosphamide etc. may be added as an immunosuppressive and steroid sparing agent ${ }^{13}$.

\section{Conclusion}

lgG4 related periaortitis is a very rare disease and it is often under-diagnosed. Undiagnosed cases and delayed diagnosis may result in sequelae and life-threatening complications like rupture of aortic aneurysms and dependant organ ischemia and dysfunction. If it remains untreated prognosis is poor. This condition can be treated adequately with the rewarding result and good prognosis if the diagnosis is made early. Diagnosis is missed or delayed due to uncommon and silent nature of the disease, slow progression, nonspecific symptoms and lack of awareness and investigation facilities. A high index of suspicion and adequate knowledge is required to diagnose and manage the patient of lgG4 related periaortitis.

\section{References}

1. Wallace ZS, Deshpande V, Mattoo H et al. IgG4-Related Disease: Clinical and Laboratory Features in One Hundred Twenty-Five Patients. Arthritis Rheumatol 2015; 67(9):2466-75.
2. Stone JH, Khosroshahi A, Deshpande V et al. Recommendations for the nomenclature of lgG4-related disease and its individual organ system manifestations. Arthritis Rheum 2012; 64(10):3061-7.

3. Deshpande V, Zen Y, Chan JKet al. Consensus statement on the pathology of lgG4-related disease. Mod Pathol 2012; 25(9):1181-92.

4. Khosroshahi A, Wallace ZS, Crowe JL et al. International Consensus Guidance Statement on the Management and Treatment of IgG4-Related Disease. Arthritis Rheumatol 2015; 67(7):1688-99.

5. ArezouKhosroshahi; John H. Stone. A clinical overview of IgG4related systemic disease. Current Opinion in Rheumatology 2011; 23(1):57-66.

6. John H. Stone; Yoh Zen; Vikram Deshpande. "IgG4-Related Disease". The New England Journal of Medicine 2012; 366(6):539-51.

7. Zeina AR, Slobodin G, Naschitz JE et al. Isolated periaortitis: clinical and imaging characteristics. Vasc Health Risk Manag 2007; 3(6):1083-6.

8. Mizushima I, Kasashima S, Fujinaga $Y$ et al. IgG4-related periaortitis/periarteritis: An under-recognized condition that is potentially life-threatening. Mod Rheumatol 2019; 29(2):240-50.

9. Mizushima I, Inoue D, Yamamoto $M$ et al. Clinical course after corticosteroid therapy in IgG4-related aortitis/periaortitis and periarteritis: a retrospective multicenter study. Arthritis Res Ther 2014; 16(4):R156.

10. Inoue D, Zen Y, Abo H, Gabata Tet al. Immunoglobulin G4-related periaortitis and periarteritis: CT findings in 17 patients. Radiology $2011 ; 261(2): 625-33$.

11. Ozawa M, Fujinaga $Y$, Asano J et al. Clinical features of IgG4related periaortitis/periarteritis based on the analysis of 179 patients with IgG4-related disease: a case-control study. Arthritis Res Ther 2017; 19:223.

12. Mavrogeni S, Markousis-Mavrogenis G, Kolovou G. IgG4related cardiovascular disease. The emerging role of cardiovascular imaging. Eur J Radiol 2017; 86:169-75.

13. Inoue D, Zen Y, Abo Het al. Immunoglobulin G4-related periaortitis and periarteritis: CT findings in 17 patients. Radiology $2011 ; 261(2): 625-33$.

14. Tajima M, Hiroi Y, Takazawa Yet al. Immunoglobulin G4-related multiple systemic aneurysms and splenic aneurysm rupture during steroid therapy. Hum Pathol 2014; 45(1):175-179. . 